\title{
Long-Term Survival After Multidisciplinary Management of Resected Pancreatic Adenocarcinoma
}

\author{
Matthew H. G. Katz, MD ${ }^{1,2}$, Huamin Wang, MD, $\mathrm{PhD}^{3}$, Jason B. Fleming, $\mathrm{MD}^{1}$, Charlotte C. Sun, DrPH, MPH ${ }^{4}$, \\ Rosa F. Hwang, MD ${ }^{1}$, Robert A. Wolff, MD ${ }^{5}$, Gauri Varadhachary, MD ${ }^{5}$, James L. Abbruzzese, MD \\ Christopher H. Crane, MD ${ }^{6}$, Sunil Krishnan, MD $^{6}$, Jean-Nicolas Vauthey, $\mathrm{MD}^{1}$, Eddie K. Abdalla, MD ${ }^{1}$, \\ Jeffrey E. Lee, MD ${ }^{1}$, Peter W. T. Pisters, MD $^{1}$, and Douglas B. Evans, MD $^{7}$ \\ ${ }^{1}$ Department of Surgical Oncology, The University of Texas M. D. Anderson Cancer Center, Houston, TX; ${ }^{2}$ Department of \\ Surgery, Division of Hepatobiliary and Pancreas Surgery, The University of California at Irvine, Orange, CA; ${ }^{3}$ Department \\ of Pathology, The University of Texas M. D. Anderson Cancer Center, Houston, TX; ${ }^{4}$ Department of Gynecologic \\ Oncology, The University of Texas M. D. Anderson Cancer Center, Houston, TX; ${ }^{5}$ Department of Gastrointestinal Medical \\ Oncology, The University of Texas M. D. Anderson Cancer Center, Houston, TX; ${ }^{6}$ Department of Radiation Oncology, \\ The University of Texas M. D. Anderson Cancer Center, Houston, TX; ${ }^{7}$ Department of Surgery, The Medical College of \\ Wisconsin, Milwaukee, WI 53226-3596
}

\begin{abstract}
Introduction. Actual 5-year survival rates of $10-18 \%$ have been reported for patients with resected pancreatic adenocarcinoma (PC), but the use of multimodality therapy was uncommon in these series. We evaluated long-term survival and patterns of recurrence in patients treated for PC with contemporary staging and multimodality therapy. Methods. We analyzed 329 consecutive patients with PC evaluated between 1990 and 2002 who underwent resection. Each received a multidisciplinary evaluation and a standard operative approach. Pre- or postoperative chemotherapy and/or chemoradiation were routine. Surgical specimens of 5-year survivors were rereviewed. A multivariate model of factors associated with long-term survival was constructed.

Results. Patients underwent pancreaticoduodenectomy $(n=302 ; 92 \%)$, distal $(n=20 ; 6 \%)$, or total pancreatectomy $(n=7 ; 2 \%)$. A total of 108 patients $(33 \%)$ underwent vascular reconstruction, 301 patients $(91 \%)$ received
\end{abstract}

Presented at the 2008 Annual Meeting of the Society of Surgical Oncology in Chicago, IL.

(C) The Author(s) 2009. This article is published with open access at Springerlink.com

First Received: 21 July 2008;

Published Online: 5 February 2009

M. H. G. Katz, MD

e-mail: katzmh@uci.edu neoadjuvant or adjuvant therapy, 157 specimens (48\%) were node positive, and margins were microscopically positive in 52 patients (16\%). Median overall survival and disease-specific survival was 23.9 and 26.5 months. Eighty-eight patients $(27 \%)$ survived a minimum of 5 years and had a median overall survival of 11 years. Of these, $21(24 \%)$ experienced recurrence, $7(8 \%)$ after 5 years. Late recurrences occurred most frequently in the lungs, the latest at 6.7 years. Multivariate analysis identified disease-negative lymph nodes $(P=.02)$ and no prior attempt at resection $(P=0.01)$ as associated with 5-year survival.

Conclusions. Our 27\% actual 5-year survival rate for patients with resected PC is superior to that previously reported, and it is influenced by our emphasis on detailed staging and patient selection, a standardized operative approach, and routine use of multimodality therapy.

Surgical resection of the involved portion of pancreas has long been characterized as the only potentially curative therapy for patients with pancreatic adenocarcinoma (PC). Although removal of the primary tumor is necessary to ensure long-term survival, it is usually not sufficient. Of the $10 \%$ of patients who seek care with anatomically localized, stage I and II PC, surgical resection alone has been associated with a median survival of only 11-20 months as a result of high rates of postoperative tumor recurrence. ${ }^{1-5}$ Such data provide support for ongoing efforts to combine surgery with systemic therapies and chemoradiation. For 
patients who have locally advanced (stage III) PC, complete resection of the primary tumor is usually not possible, and for those with metastatic (stage IV) PC, surgery directed at the primary tumor offers no clinical benefit. Therein lies the importance of accurately defining the extent of disease at the time of diagnosis and reserving surgery for those patients with localized, nonmetastatic PC who can undergo a complete gross resection of the primary tumor. ${ }^{6,7}$

For patients with resectable PC, actual rates of longterm survival and systematic analyses of clinicopathologic factors associated with it have only recently been published. ${ }^{8-14}$ Series of long-term survivors published within the past decade demonstrate that $10-18 \%$ of patients who have undergone resection for PC at major referral centers have survived at least 5 years. However, most such patients were treated in an era in which pretreatment staging was inferior to that available today, the use of adjuvant/neoadjuvant therapy was uncommon, and vascular resection and reconstruction at the time of pancreatectomy was rarely performed. ${ }^{15}$ Moreover, these series often included patients with incompletely resected locally advanced disease (grossly positive margins of resection, R2) and those with metastatic disease not appreciated on preoperative imaging, in whom the postoperative outcome is expected to be poor. ${ }^{16}$ Finally, the data presented in these reports were often incomplete, particularly with regard to the timing and pattern of disease recurrence. Taken together, these limitations make the conclusions drawn from prior studies of long-term, postsurgical survival difficult to apply to patients treated with a more modern and systematic multidisciplinary approach to their disease.

Over the past two decades, important advances in the perioperative management of patients with $\mathrm{PC}$ have led to a reduction in the morbidity and mortality associated with pancreatic surgery. In an attempt to further improve patient outcome, we and others have sought to better define who should undergo surgery with a more objective definition of the term resectable, to extend the limits of pancreaticoduodenectomy (PD) to include a standardized approach to venous resection when needed, and to emphasize the routine use of combined modality therapy. ${ }^{17-19}$

At our institution, the pretreatment staging evaluation, treatment, and follow-up of all patients with PC is coordinated by a multidisciplinary working group that meets weekly and is supported by an integrated database and tumor bank. ${ }^{20}$ Since 1990, we have used standardized definitions and algorithms for clinical/radiographic staging, surgical technique, and histopathologic analysis of surgical specimens. Recent outcome data have supported our approach to venous resection and reconstruction when necessary to achieve a gross complete $(\mathrm{R} 0, \mathrm{R} 1)$ resection. ${ }^{18}$ Finally, results from our clinical trials of neoadjuvant therapy followed by surgery has led to an institutional bias toward this approach for patients with potentially resectable (stage I and II) and borderline resectable (subgroup of stage III) PC; in the absence of neoadjuvant therapy, postoperative adjuvant therapy has been used whenever possible. $^{21-27}$ Therefore, this report reflects a relatively homogenous population of consecutive patients who have been accurately staged and treated in a uniform fashion. The long-term outcomes reported herein accurately represent the natural history of disease in patients with resectable pancreatic cancer treated with a multidisciplinary approach.

\section{PATIENTS AND METHODS}

All patients who underwent evaluation for presumed or biopsy-proven PC were treated by members of our multidisciplinary pancreatic tumor study group in a disease-sitespecific gastrointestinal outpatient center staffed by surgical oncologists, medical oncologists, and radiation oncologists. The treatment algorithm for each patient was established and coordinated by the multidisciplinary team at a weekly meeting. All clinical data were prospectively recorded in a database maintained in the Department of Surgical Oncology, and corresponding biospecimens were preserved in an integrated tumor bank. ${ }^{20}$

Clinical data of all patients who underwent evaluation for presumed or biopsy-proven PC between March 1990 and September 2002 and who underwent resection of their primary tumor were reviewed. Patients with a final diagnosis of invasive intraductal papillary mucinous neoplasm, mucinous cystadenocarcinoma, neuroendocrine carcinoma, or any other nonpancreatic periampullary adenocarcinoma were specifically excluded from analysis.

\section{Staging}

Only patients with tumors initially characterized as either resectable or borderline resectable were considered for surgery. Staging was established by using a multidetector contrast-enhanced computed tomographic (CT) scan of the abdomen and was confirmed when necessary by multidisciplinary review. Resectable pancreatic cancer was anatomically defined by the following: (1) absence of extrapancreatic disease; (2) no evidence of tumor extension to the superior mesenteric artery (SMA), celiac axis, or hepatic artery as defined by the presence of a tissue plane between the tumor and these arteries; and (3) a patent superior mesenteric-portal vein (SMPV) confluence. ${ }^{28}$ Anatomically defined borderline resectable tumors were those that demonstrated tumor abutment $\left(180^{\circ}\right.$ or less of the circumference of the vessel) of the SMA or celiac axis; 
tumor abutment or encasement $\left(>180^{\circ}\right.$ of the circumference of the vessel) of a short segment of the hepatic artery; or short-segment occlusion of the superior mesenteric vein, portal vein, or SMPV confluence that was amenable to vascular resection and reconstruction. ${ }^{17,29}$

\section{Treatment Sequencing}

For patients with resectable PC as defined above, we have had an institutional focus on the development of clinical trials exploring the use of protocol-based neoadjuvant therapy before surgical resection. ${ }^{22-24,26,27,30} \mathrm{In}$ general, patients with resectable, biopsy proven disease usually received neoadjuvant therapy either on or off protocol; if tissue confirmation of disease was not obtained, then a surgery-first strategy was used. Patients who underwent surgery first, followed by adjuvant chemoradiation and/or systemic chemotherapy, also included those patients with resectable disease who required an urgent operation and those patients who chose this treatment sequence. Although postoperative adjuvant therapy was favored in these situations, it was not successfully delivered to all such patients. ${ }^{21}$ Patients with borderline resectable anatomy, or those with findings indeterminate for distant metastases or with a marginal performance status received neoadjuvant therapy off protocol. ${ }^{17}$

Neoadjuvant chemotherapy and/or chemoradiation was administered either at our institution or under the care of the patient's referring oncologist. The specific regimens have been previously described in detail. ${ }^{22-24,30}$ Briefly, external-beam radiotherapy usually consisted of $50.4 \mathrm{~Gy}$ in 28 fractions or $30 \mathrm{~Gy}$ in 10 fractions. Concomitant chemotherapy consisted of 5-fluorouracil, paclitaxel, gemcitabine, or capecitabine at radiosensitizing doses. Chemotherapy, when delivered alone (usually before chemoradiation in the later part of the study period examined herein), consisted of gemcitabine alone or in combination. Approximately 4 to 6 weeks after the completion of all neoadjuvant treatment, patients underwent restaging evaluation that included CT and a complete physiologic assessment to determine their suitability for operation. Patients without evidence of progressive disease and who could safely undergo major abdominal surgery in the opinion of the operating surgeon and the multidisciplinary treatment group were brought to the operating room for planned resection of the primary tumor.

Pancreatic resection was performed in a standard fashion, as previously described. ${ }^{31,32}$ Tangential or segmental resection of the superior mesenteric vein, portal vein, or SMPV confluence was performed when the operating surgeon could not separate the pancreatic head or the uncinate process from these vessels without leaving gross tumor on the vessel or risking uncontrolled venotomy. When limited involvement of the common hepatic artery was identified, segmental resection of this vessel was performed with primary anastomosis or interposition grafting.

\section{Histopathologic Evaluation of Surgical Specimens}

For the purposes of this report, the original pathology report of each patient was rereviewed in detail. When available, the original histopathology slides of all patients who survived at least 5 years were rereviewed by a single faculty gastrointestinal pathologist (H.W.) to confirm the diagnosis. Pretreatment cytologic or histologic biopsy samples from patients who were reported to have experienced a complete response to neoadjuvant therapy were also rereviewed.

Since July 1990, a standardized system for the pathologic evaluation of PD specimens has been used at our institution. This system enabled prospective evaluation of the status of the SMA margin of resection. The technique for assessment of the SMA margin was the same regardless of whether vascular resection was performed. Early in our institutional experience, the SMA margin was evaluated by microscopic examination of an en face section. ${ }^{33}$ Beginning in January 2000, the SMA margin was evaluated according to the American Joint Commission on Cancer (AJCC) Cancer Staging Manual (6th edition) guidelines. ${ }^{34}$ Margins were designated R0 if no tumor cells were identified at all of the resection margins. The designation R1 was applied if tumor cells were present at the SMA margin or at the final common bile/hepatic duct or pancreatic transection margin (these later two margins were often resected again in the event of a positive frozen-section diagnosis). Tumor size was calculated by the pathologist by measuring the maximum gross transverse diameter of the tumor after resection. This measurement was difficult to determine in some patients after preoperative therapy because the tumor was often hard to distinguish from uninvolved adjacent pancreatic parenchyma by gross examination. Although histopathologic grade was recorded for each cancer, this pathologic factor was not included in the univariate or multivariate analyses because of the relatively subjective nature of the assessment of tumor grade.

\section{Follow-Up and Statistical Analysis}

After completion of all treatment, patients were evaluated every 3-4 months by physical examination, chest radiography, and abdominal CT scan. In patients without evidence of disease after 2 years of follow-up, evaluations were reduced to 6-month intervals. All but five patients evaluated during the time period under study had a minimum of 5 years of follow-up unless they died within 5 years of their date of diagnosis. The remaining five 
patients (detailed below) were foreign and were lost to follow-up within 5 years from diagnosis. These five patients were excluded from the analysis.

The development of a new low-density mass in the region of the resected pancreas or root of mesentery was considered evidence of local recurrence, even in the absence of symptoms or biopsy confirmation. Radiographic evidence of a new low-density mass in the liver or lungs was typically considered evidence for distant recurrence. Peritoneal recurrence was defined as the development of new ascites on physical examination or CT. When radiographic findings were consistent with recurrent cancer, biopsy was rarely performed except in patients with late recurrence of disease ( $>5$ years), particularly when first identified in the lung; in these patients, biopsy was often performed to rule out a second primary lung cancer. Only the first site or sites of recurrent disease was documented.

Patients who died after a confirmed recurrence were assumed to have died of PC (DWD) unless otherwise noted. Patients who died without recurrence were considered to have died of another cause (DOC), either if another cause was documented or if another cause was not documented but the patient had been found on serial evaluation to have no evidence of disease (NED) within 3 months of death. Patients in whom cause of death was not recorded and in whom time of death from last clinical follow-up exceeded 3 months were classified as having died of unknown cause. Patients alive at the last evaluation were classified either as NED or as alive with disease (AWD) if recurrence was previously documented.

All follow-up was measured from the date of histopathologic diagnosis. Overall survival was calculated by the date of death or last contact. Disease-specific survival was calculated by the date of death with disease; patients who died of another or unknown cause were censored. The Kaplan-Meier method was used to generate survival curves by clinical characteristics. Multiviariate logistic regression analysis was conducted to identify factors associated with overall survival of $\geq 5$ years. Variables that were significant on univariate analysis at $P \leq .25$ were included in the multivariate analysis. All statistical tests were two tailed, with a significance level of $P<.05$. SPSS software version 15.0 (SPSS, Chicago, IL) was used for all statistical analyses.

\section{RESULTS}

Between 1990 and 2002, a total of 2,217 patients with biopsy-confirmed PC were evaluated at our institution. Among these, the initial radiographic evaluation revealed locally advanced or metastatic disease in 610 and 992 patients, respectively. Of the remainder, 337 (15\% of 2,217 total patients) ultimately underwent surgical resection for a resectable or borderline resectable primary PC. Follow-up for survival was complete for a minimum of 5 years or to the date of death (if death occurred before 5 years) in 332 (99\%) of the 337 patients. The remaining five patients were lost to follow-up at a median of 9.8 months from diagnosis and were excluded from analysis. Each of these five patients came from outside the United States and had tumors of the pancreatic head or uncinate process. None had previously been administered chemotherapy or chemoradiation; three had undergone prior laparotomy with an unsuccessful attempt at tumor resection. At referral, one patient underwent an R0 PD and was found to have disease-negative lymph nodes. The remaining four patients received neoadjuvant treatment before PD: three underwent an R0 resection and had negative lymph nodes, one had a positive SMA margin, and four had positive regional lymph nodes. One of the five patients lost to follow-up required vascular resection at the time of PD.

Of the 332 patients whom we could evaluate, 91 (27\%) survived at least 5 years, and $241(73 \%)$ patients died within 5 years of diagnosis. Original histopathology slides of the tumors from 86 of the 91 patients who survived 5 years were available for repeat review. Pathology repeat review led to a change in the diagnosis of four $(5 \%)$ patients, including a change to adenocarcinoma arising in the distal common bile duct in two patients, adenocarcinoma arising within an intraductal papillary mucinous neoplasm in one patient, and a mixed adenocarcinoma/neuroendocrine carcinoma in one patient. This last patient was left in the study population, and the other three patients were excluded from further analysis. The original diagnosis of PC of ductal origin was confirmed in the remaining 82 patients (of 86 with available slides). After excluding 3 of the 4 patients in whom the diagnosis was changed, the final study population included 329 patients: $88(27 \%)$ who survived 5 years ( 83 of whom underwent pathology repeat review), and 241 (73\%) who did not survive 5 years. Systemic chemotherapy and/or chemoradiation had been administered to 21 patients (6\%) before referral, and 48 patients (15\%) had undergone prior laparotomy with an unsuccessful attempt at tumor resection.

Demographic and treatment factors are listed in Table 1. Univariate analysis revealed no difference between cohorts of patients who did and did not survive 5 years in terms of sex, age, type of operation performed and the need for vascular resection, operative blood loss, length of hospital stay, use of neoadjuvant or adjuvant therapy, or tumor size. However, patients who survived 5 years were less likely to have undergone attempted tumor resection before referral and were more likely to have undergone a margin-negative (R0) resection and to have disease-negative lymph nodes. Surgical margins were grossly negative in all patients; 52 (16\%) of 329 total patients were found to have microscopically positive (R1) margins in the final pathology report. 
TABLE 1 Univariate analysis of demographic and treatmentrelated factors for the 329

consecutive patients who were evaluated for pancreatic adenocarcinoma between 1990 and 2002 and underwent resection

$P$ comparison between survival cohorts

a Ratio of the number of positive lymph nodes to the number examined

b Adenocarcinoma identified in 6 of 12 nodes in one patient and 1 of 2 in the other

CHA common hepatic artery

\begin{tabular}{|c|c|c|c|c|}
\hline Clinical factor & Total & $\begin{array}{l}\text { Survival } \\
<5 \text { years }\end{array}$ & $\begin{array}{l}\text { Survival } \\
\geq 5 \text { years }\end{array}$ & $P$ value \\
\hline Patients $(n)$ & 329 & 241 & 88 & \\
\hline \multicolumn{5}{|l|}{ Demographics } \\
\hline Sex, $n(\%)$ & & & & .34 \\
\hline Male & $190(58)$ & $143(59)$ & $47(53)$ & \\
\hline Female & $139(42)$ & $98(41)$ & $41(47)$ & \\
\hline Age (years) & & & & .70 \\
\hline Median (mean) & $64(63)$ & $64(63)$ & $63(62)$ & \\
\hline Range & $30-85$ & $39-82$ & $30-85$ & \\
\hline \multicolumn{5}{|l|}{ Surgery } \\
\hline Type of operation, $\mathrm{n}(\%)$ & & & & .26 \\
\hline Pancreaticoduodenectomy & $302(92)$ & $219(91)$ & $83(94)$ & \\
\hline Distal pancreatectomy & $20(6)$ & $15(6)$ & $5(6)$ & \\
\hline Total pancreatectomy & $7(2)$ & $7(3)$ & $0(0)$ & \\
\hline Reoperative, $n(\%)$ & $48(15)$ & 43 (18) & $5(6)$ & .006 \\
\hline Vascular resection, $n(\%)$ & $108(33)$ & $83(34)$ & $25(28)$ & .30 \\
\hline Superior mesenteric or portal vein & $101(31)$ & $76(32)$ & $25(28)$ & \\
\hline CHA & $9(3)$ & $7(3)$ & $2(2)$ & \\
\hline Inferior vena cava & $4(1)$ & $4(2)$ & $0(0)$ & \\
\hline Operative blood loss (ml) & & & & .08 \\
\hline Median (mean) & $950(1289)$ & 1000 (1374) & $900(1057)$ & \\
\hline Range & $100-18,000$ & $100-18,000$ & $125-3600$ & \\
\hline Length of stay (days) & & & & .14 \\
\hline Median (mean) & $12(15)$ & $13(15)$ & $11(13)$ & \\
\hline Range & $5-108$ & $5-108$ & $6-41$ & \\
\hline \multicolumn{5}{|l|}{ Additional therapy } \\
\hline Neoadjuvant treatment, $n(\%)$ & $253(77)$ & $188(78)$ & $65(74)$ & .43 \\
\hline Chemotherapy & $36(11)$ & $27(11)$ & $9(10)$ & \\
\hline Chemoradiation & $248(76)$ & 185 (77) & $63(72)$ & \\
\hline Adjuvant treatment, $n(\%)$ & $59(18)$ & $42(18)$ & $17(20)$ & .68 \\
\hline Chemotherapy & $19(6)$ & $15(6)$ & $4(5)$ & \\
\hline Chemoradiation & $49(15)$ & $34(14)$ & $15(17)$ & \\
\hline Surgery alone, $n(\%)$ & $25(8)$ & $18(7)$ & $7(8)$ & .95 \\
\hline \multicolumn{5}{|l|}{ Tumor histopathology } \\
\hline Tumor size $(\mathrm{cm})$ & & & & .09 \\
\hline Median (mean) & $3.0(3.0)$ & $3.0(3.1)$ & $3.0(2.8)$ & \\
\hline Range & $.2-13$ & $.2-13$ & $.3-8.0$ & \\
\hline Margin status, $n(\%)$ & & & & .04 \\
\hline R0 & $277(84)$ & $197(82)$ & $80(91)$ & \\
\hline $\mathrm{R} 1$ & $52(16)$ & $44(18)$ & $8(9)$ & \\
\hline Lymph node status, $n(\%)$ & & & & .01 \\
\hline NO & $172(52)$ & $116(48)$ & $56(64)$ & \\
\hline N1 & $157(48)$ & $125(52)$ & $32(36)$ & \\
\hline Lymph node ratio, $n(\%)^{\mathrm{a}}$ & & & & .05 \\
\hline 0 & $171(52)$ & $115(48)$ & $56(64)$ & \\
\hline $0-.2$ & $93(28)$ & $72(30)$ & $21(24)$ & \\
\hline $.2-.4$ & $44(13)$ & $35(15)$ & $9(10)$ & \\
\hline$>.4$ & $20(6)$ & $18(7)$ & $2(2)^{b}$ & \\
\hline
\end{tabular}


Among 49 R1 PDs, microscopically positive margins included the SMA margin $(n=44)$, the final pancreatic transection margin $(n=10)$ and the final common bile/ hepatic duct margin $(n=2)$. In addition, one patient who underwent total pancreatectomy had a microscopically positive SMA margin, and two patients who underwent distal pancreatectomy had a microscopically positive pancreatic transection margin. The median number of lymph nodes examined in the pathology specimen was 15 (range, 0-48). In patients with node-positive disease, the median number of positive lymph nodes was 2 (range, 1-21). Importantly, only 25 (8\%) of the 329 patients underwent surgery alone without any form of additional therapy; 301 $(91 \%)$ received either preoperative or postoperative chemotherapy or chemoradiation. In three patients who did not receive neoadjuvant therapy, the administration of postoperative therapy could neither be confirmed nor excluded.

\section{Survival}

The median overall survival of the 329 patients was 23.9 months (95\% confidence interval [95\% CI] 20.89, 26.9). Eighty-eight patients survived 5 years from diagnosis for an actual 5-year survival rate of $27 \%$ (Fig. 1a). At last follow-up, 275 (84\%) of 329 patients had died, including $4(1 \%)$ who died in the perioperative period. The median follow-up for the 54 patients who were still alive at last follow-up was 7.6 years (range, 5.0-16.1 years).

The 88 patients who survived 5 years or longer had a median overall survival of 11.3 years. At last follow-up, 34 of these 5-year survivors had died and 54 remained alive. Of the 34 patients who had died, 17 were classified as DWD and 11 were classified as DOC and had no evidence of PC at the time of death. Six patients died of unknown cause. Of the 54 patients who were alive at last follow-up,
51 were NED and 3 were AWD. The 241 patients who did not survive 5 years included the 4 perioperative deaths, 208 patients DWD, 19 DOC, and 10 in whom the exact cause of death could not be determined. The disease-specific survival for the entire population was 26.5 months (95\% CI 23.1, 29.9) (Fig. 1b).

Of the 329 total patients in this analysis, 145 patients treated between 1990 and 1998 had a potential of 10 years of follow-up. Among these, 20 patients survived 10 years, yielding a 10-year actual survival rate of $14 \%$. At last contact, 10 of the 20 patients were NED, 7 were DOC, and in 3, the exact cause of death could not be determined; these 3 patients were last documented NED at 10.3 years, 11.1 years, and 11.6 years after diagnosis. No 10-year survivor died with documented recurrent $\mathrm{PC}$ at the time of death.

Treatment and histopathologic clinical factors were compared between patients who did and did not survive 5 years by a multivariate logistic regression model constructed from variables that were significant on univariate analysis at $P \leq$ .25 (Table 2). By use of this model, we found that negative lymph nodes (odds ratio 1.92, 95\% CI 1.13, 3.27, $P=.02$ ) and lack of a history of prior attempt at resection (odds ratio $4.05,95 \%$ CI $1.97,11.94, P=.01$ ) were statistically significant prognostic factors associated with 5-year survival.

\section{Disease Recurrence}

Recurrent PC was documented in 229 (70\%) of the 329 patients at 244 sites of first recurrence (Fig. 2). Of these 229 patients, 225 were DWD and 3 were AWD at last contact. One additional patient experienced recurrence but was classified as DOC at the time of death; this patient developed histopathologically confirmed metastatic PC isolated to the left frontal lobe of the brain 21 months after diagnosis, underwent craniotomy and metastasectomy, and ultimately
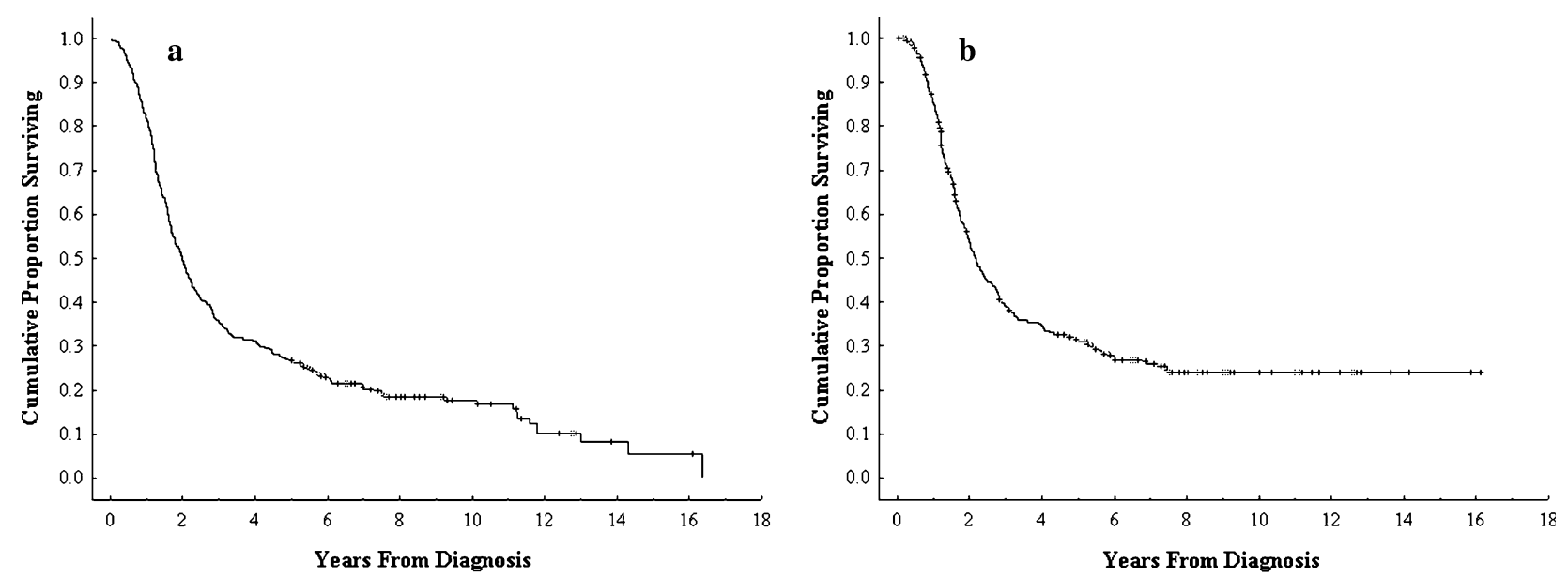

FIG. 1 (a) Actual overall and (b) disease-specific survival of patients with resectable or borderline resectable pancreatic adenocarcinoma who were evaluated at M. D. Anderson Cancer Center between 1990 and 2002 and who underwent resection 
TABLE 2 Multivariate analysis of clinicopathologic factors associated with 5-year survival

\begin{tabular}{lllll}
\hline Prognostic variable & $\begin{array}{l}\text { No. of } \\
\text { patients }\end{array}$ & $\begin{array}{l}\text { Odds } \\
\text { ratio }\end{array}$ & $95 \%$ CI & $P$ value \\
\hline No prior attempt at resection & 281 & 4.05 & $1.97,11.94$ & .01 \\
Lymph nodes negative (N0) & 157 & 1.92 & $1.13,3.27$ & .02 \\
R0 resection & 277 & 1.96 & $.86,4.45$ & .11 \\
\hline
\end{tabular}

Factors included in the multivariate model included those significant on univariate analysis at $P \leq .25$ : tumor size, length of stay, previous attempt at tumor resection, $\mathrm{R}$ status, nodal status, and $E B L$ estimated blood loss

95\% CI 95\% confidence interval

died 9.5 years after her original diagnosis. Cause of death was renal failure unrelated to her cancer. All confirmed cases of recurrence occurred within 6.7 years of diagnosis and 211 (92\%) of the 229 patients who experienced recurrence did so within the first 3 years after diagnosis. Death with disease occurred at a median of 5.6 months after the date of first recurrence (range, .1-57.1 months) in the 225 patients who died with documented recurrent PC.

Among the 229 patients whose disease recurred, the most common sites of first recurrence were liver $(n=107$; $47 \%)$, lung $(n=50 ; 22 \%)$, tumor bed and regional lymph nodes $(n=39 ; 17 \%)$, and peritoneum $(n=30 ; 13 \%)$ (Fig. 2). Recurrence within the first 3 years of diagnosis most commonly manifested as hepatic metastasis: 104 (46\%) of 226 sites of first recurrence within the first 3 years of diagnosis versus $3(17 \%)$ of 18 sites of first recurrence after 3 years from diagnosis. In contrast, recurrent disease identified after the third year was most common in the lungs: 10 (56\%) of 18 sites of first recurrence after the third year versus $40(18 \%)$ of 226 sites of first recurrence within the first 3 years.

Among the 88 patients who survived 5 years, 21 patients experienced recurrence (Table 3). Within the first 5 years of diagnosis, $14(67 \%)$ of 21 patients recurred at 15 sites: liver $(n=3 ; 21 \%)$, lung $(n=7 ; 50 \%)$, tumor bed or regional lymph nodes $(n=2 ; 14 \%)$, and other sites $(n=3 ; 21 \%)$. After 5 years of follow-up, we documented an additional seven patients with first sites of recurrent PC in the lung $(n=6 ; 86 \%)$, peritoneum $(n=1 ; 14 \%)$, or dermis $(n=1 ; 14 \%)$. For each of these late recurrences, a tissue biopsy was performed to exclude a second primary cancer. In total, of 21 patients whose disease recurred and who survived 5 years, only $3(14 \%)$ experienced recurrence recurred in the liver and only $2(10 \%)$ experienced local recurrence. Most first recurrences in the 21 patients who survived 5 years were located in the lung $(n=13$; $62 \%$ ), particularly when the recurrence developed late. In contrast, of the 208 patients who experienced recurrence and who did not survive 5 years, recurrences occurred in the liver $(n=104 ; 50 \%)$, tumor bed and regional lymph nodes $(n=38 ; 18 \%)$, lung $(n=37 ; 18 \%)$, and peritoneum $(n=28 ; 14 \%)$ (Table 4).

\section{DISCUSSION}

Of 329 consecutive patients who underwent surgical resection of their primary PC, $88(27 \%)$ survived 5 years, which to our knowledge is the highest actual survival rate reported (Table 5). We attribute this result to the use of objective criteria for the selection of patients for surgery, a standardized approach to the technical aspects of the operation (to minimize local recurrence), an institutional emphasis on multimodality therapy, and the frequent use of neoadjuvant treatment sequencing, which may result in avoiding surgery in patients most susceptible to early disease recurrence. However, it is important to note that patients included in this report were treated between 1990 and 2002 to allow for a minimum follow-up of 5 years; patients treated more recently may do even better with the progress (albeit modest) that has resulted from the
FIG. 2 Frequency, location, and timing of disease recurrence after resection for patients with resectable and borderline resectable pancreatic adenocarcinoma

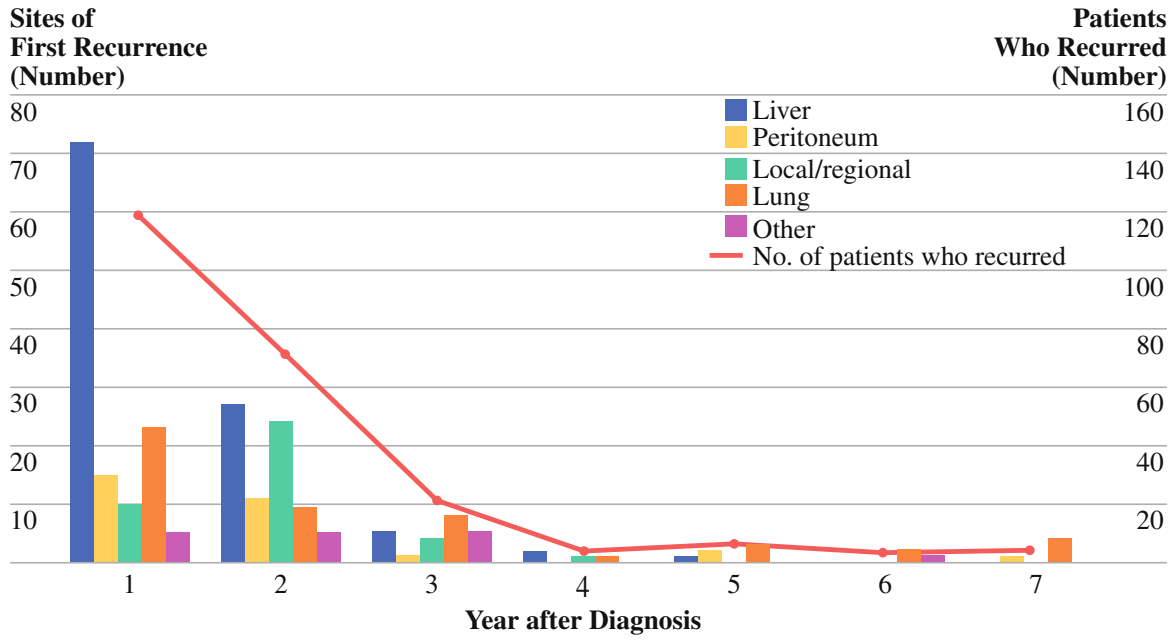


TABLE 3 Timing and first site or sites of recurrent disease in 21 five-year survivors whose disease recurred
$D W D$ dead with disease, $D O C$ dead of other cause, $A W D$ alive with disease

${ }^{a}$ Histopathologically verified

TABLE 4 Comparison of sites of first recurrence between 5year survivors and patients who did not survive 5 years

\begin{tabular}{|c|c|c|c|c|c|}
\hline Patient No. & $\begin{array}{l}\text { Site of first } \\
\text { recurrence }\end{array}$ & $\begin{array}{l}\text { Time to } \\
\text { recurrence } \\
\text { (months) }\end{array}$ & $\begin{array}{l}\text { Recurrence to } \\
\text { last follow-up } \\
\text { (months) }\end{array}$ & $\begin{array}{l}\text { Overall survival } \\
\text { (months) }\end{array}$ & $\begin{array}{l}\text { Current } \\
\text { status }\end{array}$ \\
\hline 1 & Lung $^{\mathrm{a}}$ & 10 & 54 & 64 & DWD \\
\hline 2 & Liver & 12 & 56 & 68 & DWD \\
\hline 3 & Locoregional $^{\mathrm{a}}$ & 13 & 47 & 60 & DWD \\
\hline 4 & Lung $^{\mathrm{a}}$ & 16 & 57 & 73 & DWD \\
\hline 5 & Brain $^{a}$ & 21 & 114 & 135 & DOC \\
\hline 6 & Abdominal wall ${ }^{\mathrm{a}}$ & 25 & 41 & 66 & AWD \\
\hline 7 & Liver, lung & 29 & 37 & 66 & DWD \\
\hline 8 & Liver $^{\mathrm{a}}$ & 42 & 23 & 65 & DWD \\
\hline 9 & Locoregional $^{\mathrm{a}}$ & 43 & 27 & 69 & DWD \\
\hline 10 & Lung $^{\mathrm{a}}$ & 47 & 23 & 70 & AWD \\
\hline 11 & Lung $^{\mathrm{a}}$ & 49 & 20 & 69 & DWD \\
\hline 12 & Lung $^{\mathrm{a}}$ & 57 & 9 & 65 & AWD \\
\hline 13 & Lung & 57 & 3 & 60 & DWD \\
\hline 14 & Peritoneum $^{\mathrm{a}}$ & 58 & 7 & 65 & DWD \\
\hline 15 & Lung $^{\mathrm{a}}$ & 70 & 15 & 85 & DWD \\
\hline 16 & Lung $^{a}$ & 71 & 1 & 71 & DWD \\
\hline 17 & Dermis $^{\mathrm{a}}$ & 71 & 1 & 73 & DWD \\
\hline 18 & Lung $^{\mathrm{a}}$ & 76 & 12 & 88 & DWD \\
\hline 19 & Lung $^{\mathrm{a}}$ & 78 & 12 & 91 & DWD \\
\hline 20 & Lung, ${ }^{a}$ Peritoneum & 80 & 4 & 83 & DWD \\
\hline 21 & Lung $^{a}$ & 80 & 11 & 91 & DWD \\
\hline
\end{tabular}

\begin{tabular}{lllll}
\hline Characteristic & $\begin{array}{l}\text { Survival } \\
<5 \text { years } \\
(n=241)\end{array}$ & & Survival $\geq 5$ years $(n=88)$ & \\
\cline { 3 - 5 } & $n$ & $n$ & $\begin{array}{l}\text { Recurrence } \\
<5 \text { years }(n)\end{array}$ & $\begin{array}{l}\text { Recurrence } \\
\geq 5 \text { years }(n)\end{array}$ \\
\hline No. of patients whose disease recurred & 208 & 21 & 14 & 7 \\
Site of first recurrence & & & & \\
Liver & $104(50)$ & $3(14)$ & $3(21)$ & 0 \\
Lung & $37(18)$ & $13(62)$ & $7(50)$ & $6(86)$ \\
Locoregional & $38(18)$ & $2(10)$ & $2(14)$ & 0 \\
Peritoneum & $28(13)$ & $2(10)$ & $1(7)$ & $1(14)$ \\
Abdominal wall/dermis & $1(1)$ & $2(10)$ & $1(7)$ & $1(14)$ \\
Brain & 0 & $1(5)$ & $1(7)$ & 0 \\
Bone & $3(1)$ & 0 & 0 & 0 \\
Other & $6(3)$ & 0 & 0 & 0 \\
\hline
\end{tabular}

a Totals may exceed $100 \%$ as a result of multiple sites of first recurrence in several patients resectable, borderline resectable, locally advanced, or metastatic. ${ }^{29}$ Importantly, local tumor resectability was determined preoperatively, and surgery was considered only in patients with resectable or borderline resectable disease. In the absence of extrapancreatic metastatic disease, an aggressive approach was taken to venous resection and reconstruction when isolated venous involvement was the only impediment to an otherwise complete gross resection. ${ }^{18}$ In contrast, we did not attempt resection in CT-based criteria to define the extent of disease as by experienced oncology specialists. We used objective

development and use of systemic chemotherapy and the gery into contemporary treatment schemas.

The selection of patients for surgery was influenced by the use of high-quality pretreatment cross-sectional imaging with CT, treatment sequencing that often involved neoadjuvant therapy, and the clinical assessment of patients 
TABLE 5 International series of long-term survivors after treatment for pancreatic adenocarcinoma

\begin{tabular}{|c|c|c|c|c|c|c|c|c|c|c|}
\hline Series & $\begin{array}{l}\text { No. of } \\
\text { patients }\end{array}$ & $\begin{array}{l}\mathrm{PD} \\
(\%)\end{array}$ & $\begin{array}{l}\text { Stages } \\
\text { included }\end{array}$ & $\begin{array}{l}\text { Adjuvant } \\
\text { treatment } \\
(\%)\end{array}$ & $\begin{array}{l}\mathrm{R} 0 \\
(\%)\end{array}$ & $\begin{array}{l}\mathrm{R} 1 / \mathrm{R} 2 \\
(\%)\end{array}$ & $\begin{array}{l}\mathrm{N} 1 \\
(\%)\end{array}$ & $\begin{array}{l}\text { Vascular } \\
\text { resection } \\
(\%)\end{array}$ & $\begin{array}{l}\text { Median } \\
\text { OS (Mo) }\end{array}$ & $\begin{array}{l}\text { Actual 5-year } \\
\text { survivors, } \\
n \text { (rate } \% \text { ) }\end{array}$ \\
\hline MDACC (current study) & 329 & 92 & PR, BR & 91 & 84 & $16 / 0$ & 48 & 33 & 24 & $88(27)$ \\
\hline Mayo Clinic ${ }^{13}$ & 357 & 100 & PR, LA, M & 77 & 77 & $18 / 5$ & 49 & 13 & 17 & $62(17)$ \\
\hline Johns Hopkins $^{12}$ & 564 & 100 & NR & NR & NR & NR & NR & NR & NR & $96(17)$ \\
\hline $\begin{array}{l}\text { Memorial Sloan-Kettering } \\
\text { Cancer Center }^{9}\end{array}$ & 618 & 85 & $\mathrm{PR}, \mathrm{M}$ & 21 & 73 & $27 / 0$ & NR & 0 & NR & $75(12)$ \\
\hline University of Indiana ${ }^{11}$ & 226 & 90 & PR, LA & 34 & 70 & $28 / 2$ & 56 & 6 & 13 & $9(4)$ \\
\hline Seoul National University, Korea ${ }^{10}$ & 123 & 81 & PR, LA, M & 49 & 76 & $24^{\mathrm{a}}$ & 54 & 14 & 15 & $11(9)$ \\
\hline Mount Sinai Hospital, Toronto ${ }^{8}$ & 123 & 91 & PR, LA & 21 & NR & $15^{\mathrm{b}}$ & 43 & 10 & 14 & $18(15)$ \\
\hline
\end{tabular}

$P D$ pancreaticoduodenectomy, $P R$ potentially resectable, $B R$ borderline resectable, $L A$ locally advanced, $M$ metastatic, $N R$ not reported, $O S$ overall survival, $N 1$ positive regional lymphadenopathy

${ }^{a}$ No distinction made between $\mathrm{R} 1$ and $\mathrm{R} 2$ resections

${ }^{\mathrm{b}}$ No distinction made between R1 and R2 resections; number of R0 resections not stated

patients with locally advanced, stage III disease in whom a gross (R0 or R1) resection was not possible. In patients who undergo an incomplete gross (R2) resection, length of survival is not improved in comparison to cases of stage III disease managed without surgery in patients who receive chemotherapy and/or chemoradiation. ${ }^{35}$ Indeed, among patients with advanced disease $\mathrm{e}^{8-11,13}$ and among those who underwent a grossly incomplete (R2) resection ${ }^{11,13}$ who were explicitly included in prior reports of long-term survival after pancreatic resection, only a single 5-year survivor (reported to have had stage III disease) was identified. ${ }^{8}$ Accurate pretreatment staging and multidisciplinary consensus with regard to resectability (based on CT images) is something that can — and should-be performed at all medical centers that offer surgical treatment for PC. ${ }^{36}$ Such a practice will minimize the inappropriate use of surgery in patients with advanced disease and will ensure that all patients with resectable disease are considered for surgery, we hope as part of a multimodality treatment program.

Consensus is growing that chemotherapy with or without radiotherapy combined with surgery results in higher rates of survival in patients with resectable PC than surgery alone..$^{2,3,15,37-39}$ In this report, $91 \%$ of patients received either neoadjuvant or adjuvant therapy, and most patients were enrolled onto a clinical trial. Importantly, neoadjuvant therapy was delivered to $77 \%$ of all patients. The cytotoxic effect of such induction therapy is likely responsible for the lower-than-expected rates of microscopically involved lymph nodes and positive resection margins observed in the surgical specimens. ${ }^{30,40}$ The administration of chemotherapy and/or chemoradiation before surgery also enriches the population of patients selected to undergo pancreatic resection with those most likely to have a favorable outcome compared with patients treated with surgery first. In our most recent two clinical trials of neoadjuvant therapy for patients with stage I and II PC, 30\% of patients who began neoadjuvant therapy did not undergo PD as a result of disease progression, evolving medical comorbidities, or a decline in performance status. ${ }^{26,27}$ These patients, who had a median survival of $<12$ months, include those patients who would not have received a survival benefit from surgery as a result of the presence of metastatic disease that was radiographically occult at the time of pretreatment staging, and those with medical comorbidities that were also not readily apparent at the time of diagnosis. Neoadjuvant treatment sequencing accurately identified those patients who would otherwise have experienced early postoperative recurrence and those who may have an increased risk for perioperative complications; such patients did not undergo pancreatectomy. Surgery-last treatment sequencing benefits both the patients who undergo resection of their primary tumor and those who do not.

We recently reported our institutional experience with patients having borderline resectable pancreatic cancer, a prospectively identified subset of AJCC stage III disease, in whom a neoadjuvant strategy is particularly important. ${ }^{17,29}$ At our institution, three clearly defined groups of patients were classified as borderline resectable: (1) patients with strictly defined borderline resectable local tumor anatomy, predominantly arterial abutment, as described above; (2) patients with localized tumors who also had additional CT findings suspicious but indeterminate for metastatic disease; and (3) patients with a marginal but potentially recoverable performance status or extensive comorbidities requiring a prolonged evaluation that precluded immediate major abdominal surgery. In each of these three groups, we took advantage of the theoretical benefits of neoadjuvant treatment sequencing over a long time period (minimum of 4 months) of systemic chemotherapy and chemoradiation. 
Serial restaging evaluations at 2-month intervals selected patients for additional therapy and ultimately surgery; only patients with an acceptable performance status and no evidence of disease progression at the end of neoadjuvant therapy underwent surgery.

In our previously published series of 160 patients treated with this approach, $41 \%$ of patients completed all therapy, including surgery; $56 \%$ of resected specimens had a pathologic response to treatment characterized by $<50 \%$ tumor viability, and $94 \%$ of resections resulted in microscopically negative (R0) margins. The $41 \%$ of patients who completed all therapy including surgery had a median survival of 40 months. Median survival was 13 months for the $59 \%$ of patients who developed disease progression or evolving medical comorbidities. Patients with borderline resectable disease are at high risk for having CT-occult distant metastases, likely are at higher risk for a positive resection margin than patients with stage I or II PC, and often require a larger, more complex, and higher-risk operation to remove their primary tumor. When the magnitude of the operation and the risk for recurrence are both increased, neoadjuvant treatment sequencing becomes more attractive as a strategy to more precisely select patients who will benefit from pancreatectomy.

Patients who did not receive neoadjuvant therapy were routinely encouraged to receive postoperative adjuvant therapy, either, typically as part of a clinical trial. However, a number of factors may delay or prohibit the administration of adjuvant therapy in the postoperative patient. Indeed, a recent investigation of national practice patterns for multimodality therapy use reported that $<50 \%$ of patients who underwent pancreatectomy at high-volume centers received chemotherapy or chemoradiation, although the reasons for the low rate of multimodality therapy were unclear. ${ }^{15}$ These data are supported by two recently published large singleinstitution experiences in which adjuvant therapy was received by only $44 \%$ and $60 \%$ of eligible patients who had received a surgery-first treatment strategy for $\mathrm{PC}^{41,42}$ In a previous study from our institution, we reported that $26 \%$ of patients who underwent PD without any prior treatment did not receive intended adjuvant therapy. ${ }^{21}$ Patients did not receive postoperative adjuvant therapy for reasons that could be classified into three categories: treatment related (surgical complications, delayed recovery), disease related (disease progression), and patient related (advanced age, marginal preoperative performance status, medical comorbidities, patient refusal). For those patients with an Eastern Cooperative Oncology Group (ECOG) performance status that we would consider of marginal acceptance for major surgery (in the lower range of ECOG 2), a surgery-first strategy is likely to be associated with both an increased risk for complications and a high likelihood of not receiving any additional oncologic treatment. An understanding of the complexity of delivering multimodality therapy to patients of advanced age who require a large cancer operation and often have marked medical comorbidities will become even more important as our systemic therapies for PC improve.

The present report failed to reveal an association between the administration of neoadjuvant or adjuvant therapy and long-term survival. However, this is not surprising, given the high rate of combined modality treatment among all patients, the relatively modest patient numbers, and the retrospective design of this study. Only $7 \%$ of patients did not receive systemic therapy and/or chemoradiation in addition to surgery.

The frequent use of neoadjuvant therapy and the standardized approach to surgery likely influenced the low rate of margin positivity (16\%). In a previous report from our institution focusing specifically on the incidence of R1 resections and the implications of this pathologic finding, the frequency of a positive SMA margin was less in those patients who received preoperative chemoradiation (13\%) compared with those patients taken directly to surgery (19\%). ${ }^{19}$ This difference was not statistically significant but was clinically important, given the selection bias in favor of going straight to surgery, without neoadjuvant therapy, in patients with smaller tumors whose theoretical risk for an R1 resection was thought to be low. In fact, such carefully selected patients actually had a higher frequency of having a disease-positive SMA margin on final pathologic analysis of the PD specimen. This finding lends further support to the observation that PC can extend along visceral perineural fibers to the SMA and a positive microscopic margin can be found when the grossly visible tumor seems to be separate from the lateral wall of the SMA. Both multimodality therapy and proper surgical technique may be necessary to minimize the risk for a positive SMA margin and thereby reduce the risk of local recurrence. For example, all tissue to the right of the SMA should be removed with the PD specimen; the application of a stapling devise or a series of clamps without visible identification of the SMA should be avoided. In this report, local recurrence was seen radiographically in 42 (13\%) of the 329 patients. Local control is clearly necessary (but not sufficient) for long-term survival, and as our systemic therapies improve, the importance of maximizing local disease control at the time of initial treatment may provide further enthusiasm for neoadjuvant chemoradiation.

In this report, factors adversely associated with longterm survival on multivariate analysis included the presence of metastatic disease in regional lymph nodes and a previous attempt at tumor resection before referral to our institution. Of the 88 five-year survivors, only five patients had undergone an unsuccessful attempt at tumor removal before referral. However, 48 patients had undergone laparotomy for planned pancreatectomy before referral; the 
effect of an unsuccessful prereferral laparotomy was profound and something not widely discussed in the literature. The importance of carefully selecting patients for PC surgery who are compatible with the skill set of the surgeon cannot be overstated.

Of the 88 five-year survivors, $32(36 \%)$ were found to have pathologically involved lymph nodes. In fact, of the 157 node-positive patients, $32(20 \%)$ survived 5 years. To what degree the favorable outcome in patients with $\mathrm{N} 1$ disease is related to the use of multimodality therapy cannot be accurately determined. One would assume that the selection bias introduced by neoadjuvant treatment sequencing combined with the frequent use of systemic chemotherapy is partly responsible for this encouraging result. In any event, the finding that $20 \%$ of node-positive patients were alive at 5 years after diagnosis would argue against routine lymph node biopsy at the time of surgery because node-positive disease does not seem to represent a contraindication to surgical resection of the primary tumor when performed as part of a multimodal treatment strategy.

Finally, the incidence and pattern of disease recurrence warrants further comment. As demonstrated in Fig. 2, most recurrences occurred early and developed in the abdomen (largely in the liver). In contrast, late recurrences developed predominantly in the lung. This pattern of recurrence supports the use of abdominal CT or magnetic resonance imaging for routine follow-up, particularly within the first 3 to 5 years after definitive treatment. Although not analyzed in this report, we found that serum levels of CA 19-9 are frequently also helpful, unless undetectable (indicative of individuals with the Lewis $\mathrm{a}-\mathrm{b}$ blood group antigen who do not synthesize CA 19-9). In this report, the latest cancer recurrence was identified 6.7 years from diagnosis, and the latest cancer-related death occurred at 7.6 years. These findings support prior observations that 5-year survival does not always equate with cure for patients with this disease. ${ }^{9,13}$ However, the plateau of our disease-specific survival curve (Fig. 1b) does suggest that patients free of disease at 7 to 8 years after diagnosis are at very low risk for death from recurrent PC. For patients who died of recurrent disease within 5 years, death occurred at a median of 5.5 months (range, .1-36.5 months) after recurrence was identified. In contrast, of the 17 five-year survivors who died of recurrent PC, death occurred at a median of 15 months (range, .7-51 months) after the diagnosis of recurrence (Table 3). These data suggest that 5-year survivors who are not cured are characterized by an as-yet undefined tumor biology that includes a predisposition for pulmonary metastases and a more indolent form of disease progression.

In conclusion, we report a consecutive series of patients with resectable (stage I, II) and borderline resectable (stage III) PC who were treated with curative intent to include surgical resection of the primary tumor. The favorable long-term survival reported herein reflects what is possible when a well-selected group of patients are carefully staged with high-quality imaging and treated in a disease sitespecific multidisciplinary program with state-of-the-art surgery, combination chemotherapy, and radiotherapy. Future progress in the treatment of patients with PC will emphasize both the optimization of our current technologies and treatments, as well as the development of novel and more effective systemic therapies.

ACKNOWLEDGMENTS We thank Henry F. Gomez, MD, for database support, Karen R. Cleary, MD, for seminal contributions to the pathologic evaluation of surgical specimens, Chusilp Charnsangavej, MD, for support and leadership in diagnostic imaging, Jeffrey $\mathrm{H}$. Lee, MD, for program development in interventional gastroenterology, Michael J. Wallace, MD, and his colleagues in interventional radiology for management of our surgical complications, and the many patients who have entrusted the University of Texas M. D. Anderson Cancer Center with their care. Supported by the Hamill Foundation, the Various Donor Fund for Pancreatic Cancer Research, and National Institutes of Health Grant CA10193601 (SPORE in Pancreatic Cancer) at The University of Texas M. D. Anderson Cancer Center.

Open Access This article is distributed under the terms of the Creative Commons Attribution Noncommercial License which permits any noncommercial use, distribution, and reproduction in any medium, provided the original author(s) and source are credited.

\section{REFERENCES}

1. Jemal A, Siegel R, Ward E, et al. Cancer statistics, 2007. $C A$ Cancer J Clin. 2007;57:43-66.

2. Kalser MH, Ellenberg SS. Pancreatic cancer. Adjuvant combined radiation and chemotherapy following curative resection. Arch Surg. 1985;120:899-903.

3. Oettle H, Post S, Neuhaus P, et al. Adjuvant chemotherapy with gemcitabine vs observation in patients undergoing curative-intent resection of pancreatic cancer: a randomized controlled trial. JAMA. 2007;297:267-77.

4. Klinkenbijl JH, Jeekel J, Sahmoud T, et al. Adjuvant radiotherapy and 5-fluorouracil after curative resection of cancer of the pancreas and periampullary region: phase III trial of the EORTC gastrointestinal tract cancer cooperative group. Ann Surg. 1999;230:776-82.

5. Smeenk HG, van Eijck $\mathrm{CH}$, Hop WC, et al. Long-term survival and metastatic pattern of pancreatic and periampullary cancer after adjuvant chemoradiation or observation: long-term results of EORTC trial 40891. Ann Surg. 2007;246:734-40.

6. Von Hoff DD, Evans DB, Hruban RH. The pancreas. Sudbury, MA: Jones and Bartlett; 2005.

7. Sener SF, Fremgen A, Menck HR, Winchester DP. Pancreatic cancer: a report of treatment and survival trends for 100,313 patients diagnosed from 1985-1995, using the National Cancer Database. J Am Coll Surg. 1999;189:1-7.

8. Cleary SP, Gryfe R, Guindi M, et al. Prognostic factors in resected pancreatic adenocarcinoma: analysis of actual 5-year survivors. J Am Coll Surg. 2004;198:722-31.

9. Ferrone CR, Brennan MF, Gonen M, et al. Pancreatic adenocarcinoma: the actual 5-year survivors. J Gastrointest Surg. 2008;12:701-6. 
10. Han SS, Jang JY, Kim SW, et al. Analysis of long-term survivors after surgical resection for pancreatic cancer. Pancreas. 2006; 32:271-5.

11. Howard TJ, Krug JE, Yu J, et al. A margin-negative R0 resection accomplished with minimal postoperative complications is the surgeon's contribution to long-term survival in pancreatic cancer. J Gastrointest Surg. 2006;10:1338-45.

12. Riall TS, Cameron JL, Lillemoe KD, et al. Resected periampullary adenocarcinoma: 5-year survivors and their 6- to 10-year follow-up. Surgery. 2006;140:764-72.

13. Schnelldorfer T, Ware AL, Sarr MG, et al. Long-term survival after pancreatoduodenectomy for pancreatic adenocarcinoma: is cure possible? Ann Surg. 2008;247:456-62.

14. Allison DC, Piantadosi S, Hruban RH, et al. DNA content and other factors associated with ten-year survival after resection of pancreatic carcinoma. J Surg Oncol. 1998;67:151-9.

15. Bilimoria KY, Bentrem DJ, Ko CY, et al. Multimodality therapy for pancreatic cancer in the US: utilization, outcomes, and the effect of hospital volume. Cancer. 2007;110:1227-34.

16. Wolff RA, Varadhachary GR, Evans DB. Adjuvant therapy for adenocarcinoma of the pancreas: analysis of reported trials and recommendations for future progress. Ann Surg Oncol. 2008; 15:2773-86.

17. Katz MH, Pisters PW, Evans DB, et al. Borderline resectable pancreatic cancer: the importance of this emerging stage of disease. J Am Coll Surg. 2008;206:833-46.

18. Tseng JF, Raut CP, Lee JE, et al. Pancreaticoduodenectomy with vascular resection: margin status and survival duration. J Gastrointest Surg. 2004;8:935-49.

19. Raut CP, Tseng JF, Sun CC, et al. Impact of resection status on pattern of failure and survival after pancreaticoduodenectomy for pancreatic adenocarcinoma. Ann Surg. 2007;246:52-60.

20. Hwang RF, Wang H, Lara A, et al. Development of an integrated biospecimen bank and multidisciplinary clinical database for pancreatic cancer. Ann Surg Oncol. 2008;15:1356-66.

21. Aloia TA, Lee JE, Vauthey JN, et al. Delayed recovery after pancreaticoduodenectomy: a major factor impairing the delivery of adjuvant therapy? J Am Coll Surg. 2007;204:347-55.

22. Evans DB, Rich TA, Byrd DR, et al. Preoperative chemoradiation and pancreaticoduodenectomy for adenocarcinoma of the pancreas. Arch Surg. 1992;127:1335-9.

23. Staley CA, Lee JE, Cleary KR, et al. Preoperative chemoradiation, pancreaticoduodenectomy, and intraoperative radiation therapy for adenocarcinoma of the pancreatic head. Am J Surg. 1996;171:118-24.

24. Pisters PW, Abbruzzese JL, Janjan NA, et al. Rapid-fractionation preoperative chemoradiation, pancreaticoduodenectomy, and intraoperative radiation therapy for resectable pancreatic adenocarcinoma. J Clin Oncol. 1998;16:3843-50.

25. Cusack JC Jr, Fuhrman GM, Lee JE, Evans DB. Managing unsuspected tumor invasion of the superior mesenteric-portal venous confluence during pancreaticoduodenectomy. Am J Surg. 1994;168:352-4.

26. Evans DB, Varadhachary GR, Crane $\mathrm{CH}$, et al. Preoperative gemcitabine-based chemoradiation for patients with resectable adenocarcinoma of the pancreatic head. J Clin Oncol. 2008; 26:3496-502.
27. Varadhachary GR, Wolff RA, Crane $\mathrm{CH}$, et al. Preoperative gemcitabine and cisplatin followed by gemcitabine-based chemoradiation for resectable adenocarcinoma of the pancreatic head. J Clin Oncol. 2008;26:3487-95.

28. Katz MH, Hwang R, Fleming JB, Evans DB. Tumor-nodemetastasis staging of pancreatic adenocarcinoma. CA Cancer $J$ Clin. 2008;58:111-25.

29. Varadhachary GR, Tamm EP, Abbruzzese JL, et al. Borderline resectable pancreatic cancer: definitions, management, and role of preoperative therapy. Ann Surg Oncol. 2006;13:1035-46.

30. Breslin TM, Hess KR, Harbison DB, et al. Neoadjuvant chemoradiotherapy for adenocarcinoma of the pancreas: treatment variables and survival duration. Ann Surg Oncol. 2001;8:123-32.

31. Yen TW, Abdalla EK, Pisters PW, Evans DB. Pancreaticoduodenectomy. In: Von Hoff DD, Evans DB, Hruban RH, editors. Pancreatic cancer. Sudbury, MA: Jones and Bartlett; 2005. p. 265-86.

32. Gallagher SF, Zervos EE, Murr MM. Distal pancreatectomy. In: Von Hoff DD, Evans DB, Hruban RH, editors. Pancreatic cancer. Sudbury, MA: Jones and Bartlett; 2005. p. 299-312.

33. Staley CA, Cleary KR, Abbruzzese JL, et al. The need for standardized pathologic staging of pancreaticoduodenectomy specimens. Pancreas. 1996;12:373-80.

34. Exocrine pancreas. In: Greene FL, Page DL, Fleming ID, et al., editors. AJCC cancer staging manual. Chicago: Springer; 2002. p. 157-64.

35. Nitecki SS, Sarr MG, Colby TV, van Heerden JA. Long-term survival after resection for ductal adenocarcinoma of the pancreas. Is it really improving? Ann Surg. 1995;221:59-66.

36. Evans DB, Crane CH, Charnsangavej C, Wolff RA. The added value of multidisciplinary care for patients with pancreatic cancer. Ann Surg Oncol. 2008;15:2078-80.

37. Lim JE, Chien MW, Earle CC. Prognostic factors following curative resection for pancreatic adenocarcinoma: a populationbased, linked database analysis of 396 patients. Ann Surg. 2003;237:74-85.

38. Neoptolemos JP, Stocken DD, Friess H, et al. A randomized trial of chemoradiotherapy and chemotherapy after resection of pancreatic cancer. $N$ Engl J Med. 2004;350:1200-10.

39. Pawlik TM, Laheru D, Hruban RH, et al. Evaluating the impact of a single-day multidisciplinary clinic on the management of pancreatic cancer. Ann Surg Oncol. 2008;15:2081-8.

40. Pingpank JF, Hoffman JP, Ross EA, et al. Effect of preoperative chemoradiotherapy on surgical margin status of resected adenocarcinoma of the head of the pancreas. J Gastrointest Surg. 2001;5:121-30.

41. Corsini MM, Miller RC, Haddock MG, et al. Adjuvant radiotherapy and chemotherapy for pancreatic carcinoma: the Mayo Clinic experience (1975-2005). J Clin Oncol. 2008;26: 3511-6.

42. Herman JM, Swartz MJ, Hsu CC, et al. Analysis of fluorouracilbased adjuvant chemotherapy and radiation after pancreaticoduodenectomy for ductal adenocarcinoma of the pancreas: results of a large, prospectively collected database at the Johns Hopkins Hospital. J Clin Oncol. 2008;26:3503-10. 